\title{
Circuit
}

Musiques contemporaines

\section{Ultime édition de Rien à voir}

\section{Entretien avec Francis Dhomont sur l'avenir acousmatique}

\section{Maxime McKinley}

Volume 16, numéro 3, 2006

À musique contemporaine, supports contemporains?

URI : https://id.erudit.org/iderudit/902419ar

DOI : https://doi.org/10.7202/902419ar

Aller au sommaire du numéro

Éditeur(s)

Les Presses de l'Université de Montréal

ISSN

1183-1693 (imprimé)

1488-9692 (numérique)

Découvrir la revue

Citer ce document

McKinley, M. (2006). Ultime édition de Rien à voir : entretien avec Francis

Dhomont sur l'avenir acousmatique. Circuit, 16(3), 119-124.

https://doi.org/10.7202/902419ar d'utilisation que vous pouvez consulter en ligne.

https://apropos.erudit.org/fr/usagers/politique-dutilisation/ 


\title{
Ultime édition de Rien à voir Entretien avec Francis Dhomont sur l'avenir acousmatique
}

\author{
par Maxime McKinley
}

C'était en février 2004. La société de concerts Réseaux des arts médiatiques avait annoncé que la quinzième édition de ses séries de concerts Rien à voir serait la dernière de ses productions à porter ce nom. Dans le texte de présentation des notes de programme, les organisateurs ont expliqué que

lorsque nous avons démarré cette série de concerts en 1997, nous avions investi un créneau, celui des musiques acousmatiques, laissé vacant depuis plusieurs années sur la scène musicale montréalaise. On ne savait rien du succès potentiel de cette formule et notre idée n'était certes pas, à ce momentlà, de la reprendre saison après saison. Quinze éditions plus tard, après sept années d'existence, la série Rien à voir aligne des statistiques impressionnantes. [...] Mais une série régulière, c'est aussi une formule qui finit avec les années par s'épuiser. Non pas que cette musique ne nous intéresse plus, bien au contraire, mais la formule actuelle ne convient plus à la diversité des pratiques musicales d'aujourd'hui. [...] L'électro pour nous se conjugue désormais sous toutes ses déclinaisons - acousmatique, mixte, en direct, installation, etc. - et les lieux de représentation seront désormais aussi diversifiés que les pratiques elles-mêmes.

Le compositeur Francis Dhomont, un pionnier et l'un des théoriciens importants de l'art acousmatique, réagit dans l'entretien qui suit à la décision de Réseaux de mettre un terme à cette série, de même que sur la situation de l'acousmatique en général. Certes, Dhomont (né en 1926 à Paris) n'a pas manqué, tout au long de sa carrière, de s'exprimer pour défendre l'art acousmatique et l'héritage de Pierre Schaeffer. On ne sera donc pas surpris qu'il " regrette le choix fait par les responsables de Réseaux", bien qu'il maintienne tout à fait sa "confiance" et son "estime" envers le travail des animateurs de la société de concerts. Cette entrevue a été réalisée en juin 2004, par correspondance.

Maxime McKinley : Réseaux est l'un des principaux diffuseurs de musique acousmatique. Or, depuis quelque temps, la musique acousmatique ne semble plus être au cœur de sa programmation. Il se tourne de plus en plus vers la diffusion d'œuvres multidisciplinaires, d'installations, utilisant le direct, etc. Est-ce un signe que l'approche acousmatique (au sens classique ou, si l'on veut, au sens schaefferien) vit ses derniers moments de cristallisation, avant d'être reléguée définitivement à I'histoire? 
Francis Dhomont : C'est effectivement l'impression fausse et défaitiste que risque de donner cette décision. C'est, en tout cas, ce que s'empressent déjà d'en déduire les adversaires de la musique acousmatique. Pourtant, même si, en l'occurrence, je regrette le choix fait par les responsables de Réseaux, il ne fait aucun doute pour moi que c'est dans le but de renouveler et d'enrichir leurs programmes. Depuis dix ans, ils ont accompli un travail remarquable et se sont affirmés comme les seuls représentants authentiques en Amérique du Nord de l'art acousmatique. Robert Normandeau avait d'ailleurs pris les devants dès 1989 en réalisant pour l'ACREQ les concerts spatialisés du Planétarium de Montréal. Pour cela, ils sont connus et respectés internationalement. C'est précisément en raison de cet acquis incontesté qu'il me paraît inopportun de quitter la route aujourd'hui, à un moment où - dans le contexte montréalais - elle semble provisoirement plus étroite, pour s'engager dans une voie plus large mais déjà très fréquentée. Néanmoins je comprends leur point de vue et ils connaissent le mien. Et ils savent aussi l'estime que j'ai pour eux.

Alors, quand je dis "impression fausse et défaitiste" c'est que, si symptôme il y a d'un quelconque épuisement de l'approche acousmatique, il n'est ressenti qu'à Montréal, qui fut pourtant l'un des points forts de cette modalité électroacoustique. Car c'est loin d'être le cas général. On sait, notamment, que la création acousmatique est particulièrement vivante et bien représentée au Royaume-Uni. De son côté, la France, qui en est le berceau, connaît un regain très fort de concerts et de stages, comme en atteste l'intense activité de l'Association Motus, par exemple, qui organise plusieurs événements chaque mois en plus de "Futura", son festival international d'Art acousmatique annuel. II faut noter aussi l'apparition de nouveaux festivals acousmatiques, tels "Licences" ou "Elektrophonie", qui viennent s'ajouter à ceux des nombreux centres plus anciens répartis sur tout le territoire. Certains plus prestigieux, comme ceux de Bourges ou du GRM de Paris, inscrivent chaque année une quantité importante d'œuvres acousmatiques à leurs programmes. En Belgique, Musiques \& Recherches, dont le festival acousmatique international "L'espace du son" existe depuis 1984, offre maintenant des concerts mensuels à Bruxelles. En Allemagne, le secteur musicologique de l'Université de Cologne met depuis quelques années l'accent sur la production acousmatique, invite les principaux représentants de cette discipline à donner des concerts, publie des ouvrages et propose un symposium pour octobre 2004 ; le très solide ZKM Zentrum de Karlsruhe prépare actuellement un festival pour février 2005; le Luxembourg organise concerts et tables rondes en novembre prochain, etc. Ce sont quelques exemples parmi d'autres. Alors parler des "derniers moments" de la musique acousmatique avant son inhumation définitive dans l'Histoire ne paraît pas d'actualité et relève d'une approche lacunaire de la situation réelle.

Maxime McKinley : En 2003, Jean Piché a écrit : "Pour moi, la composition électroacoustique sur support a atteint son apogée entre 1990 et 1995 avec les œuvres de Francis Dhomont et de certains de ses collègues. Depuis ce temps, la pratique classique de l'électroacoustique retient difficilement mon attention'. " Croyez-vous que depuis 1995, ainsi que l'on pourrait interpréter ces 
propos de Jean Piché, la musique acousmatique est dans un état de stagnation?

Francis Dhomont : Il s'agit surtout, là encore, d'une opinion qui ne tient compte que du seul espace montréalais. L'approche acousmatique existait ailleurs bien avant la période que cite Jean Piché et, comme je crois l'avoir démontré plus haut, se poursuit bien après. Le phénomène acousmatique québécois, pour important qu'il soit, n'est qu'un chapitre d'une histoire habitée par d'autres personnages.

Et si même on se limite à la production acousmatique montréalaise, j'aimerais qu'on m'explique ce qu'il faut entendre par "stagnation". Bach a fait du Bach toute sa vie, Mozart du Mozart ou Ravel du Ravel. S'agissait-il de stagnation? Une pensée, une langue, un concept cohérents ne sont pas si vite épuisés : Bach, Mozart, Ravel utilisaient le système tonal qui est encore aujourd'hui celui de bien des œuvres occidentales, notamment de la plupart des musiques populaires. Stagnation? Depuis des siècles et à toutes les époques, les écrivains d'un même pays se servent de la même langue, progressivement évoluante, pour écrire des chefs-d'œuvre originaux. Stagnation? De la même façon, la musique acousmatique est un mode de pensée, pas une mode musicale. C'est ce que j'ai tenté d'illustrer dans une œuvre, le cycle du son, basée sur une étude de Pierre Schaeffer de 1959 : l'essence et les principes de la musique concrète y sont évidents sous une écriture électroacoustique qui fait appel aux sonorités et aux technologies les plus récentes.

Le mythe qui consiste à n'accorder de crédit qu'à la tendance en vogue pour la seule raison qu'elle est la dernière en date est une erreur aussi grave que de s'enfermer dans le passé pour la raison inverse. C'est un conditionnement de consommateur qui est né au milieu du siècle dernier avec l'industrie de l'objet jetable et dont la fugacité des systèmes informatiques actuels constitue une sorte d'apothéose. Ce qui peut, à la rigueur, être justifié pour la technologie et le commerce a contaminé le domaine de la pensée et celui de l'art. C'est ainsi qu'on assiste sur les forums Internet de musique électroacoustique à des querelles récurrentes sur l'académisme, car on est toujours académique pour quelqu'un. Tout modèle qui perdure est académique, précisément parce qu'il est devenu un modèle; il doit donc être abandonné au nom de l'inédit. L'audace d'hier cesse d'être audacieuse parce qu'elle ne choque plus, elle est intégrée et devient un nouveau classicisme. Mais, après tout, n'est-ce pas le but poursuivi? Les époques classiques constituent un aboutissement, une maturité, et ne sont généralement pas réputées pour leur médiocrité. "L'essence du classicisme est de venir après", dit Valéry. Ce qui ne signifie pas immobilisme. Car ainsi va la musique depuis ses origines, de la rupture à la stabilité et inversement. Mais à l'âge du gaspillage et de la surconsommation, on ne s'étonnera pas que les nouveautés aient la vie courte.

Et puis, l'Amérique du Nord a une propension à parer la chose nouvelle de toutes les vertus, postulant que ce qui est nouveau ne peut être que meilleur. "Ce qui fait la grandeur de l'Amérique du Nord, écrivait une agence de publicité en 1955, c'est la création de besoins et de désirs, la création du dégoût pour tout ce qui est vieux et démodé." On ne saurait mieux dire. En détruisant 
ses buildings avant qu'ils aient cent ans, l'Amérique aura-t-elle un jour un passé? D'ailleurs, le souhaite-telle? Une telle fascination pour le futur trouve son application artistique dans un besoin compulsif de tentatives éphémères, quitte à rester à la surface des choses au lieu de prendre le temps d'en explorer les profondeurs. À l'inverse, il me semble plus créatif de "travailler son instrument", comme disait le père de Schaeffer, au lieu de sauter d'une mode à l'autre en négligeant l'essentiel, c'est-à-dire le lent questionnement de soi. "Le rôle des artistes de notre époque n'est plus d'être d'avant-garde, terme militaire. Le rôle des artistes est au contraire d'être des "résistants", de construire sur ce qui a été détruit, de faire marche arrière avec les machines pour retrouver la passion, la spiritualité", estimait François Bayle en 2000 lors d'une conférence à Cologne. Si c'est cela, la stagnation, alors nous n'avons pas à en rougir.

Maxime McKinley : II semble que pour certains, "musique électroacoustique de concert" rime avec "esthétique acousmatique". Ainsi, lorsque la musique acousmatique est remise en question, bien souvent, c'est en réalité la notion du "concert classique électroacoustique" qui est remise en cause. Qu'en pensez-vous?

Francis Dhomont : Il est clair que le concert acousmatique perpétue la tradition de ce que vous nommez le "concert classique électroacoustique", pour la simple raison que c'était, dès l'origine, sous cette forme que se manifestait la musique nouvelle. Mais il y a belle lurette que la musique électroacoustique cherche à s'évader du cadre traditionnel dévolu aux concerts classiques, qu'il s'agisse du lieu, de la présentation, de l'accueil, du décor ou du cérémonial. La musique contemporaine instrumentale en fait d'ailleurs autant. Cela répond essentiellement à la quête d'un public que dissuadent souvent la nature expérimentale des œuvres et leur présentation sévère. On essaye alors de désacraliser le concert électroacoustique afin de le faire apparaître comme convivial et accessible à tous. Certaines initiatives se révèlent équilibrées, le festival Rien à voir était de celles-là. D'autres le sont moins. À trop vouloir plaire, le risque est effectivement d'évacuer le "concert classique" à force de le nier en le donnant pour autre qu'il n'est. C'est ce qui se passe lorsqu'on s'inspire des méthodes marketing pour présenter l'électroacoustique comme un divertissement sympa, festif, un événement à sensation. On espère attirer ainsi un plus large public en trouvant des titres accrocheurs, en offrant des gadgets, des boissons, en faisant rire, en étant "relax", branchés, ludiques. Mais cela ne suffit pas et, pour que le succès soit vraiment assuré, il faut aussi modifier le contenu, remplacer des œuvres, un peu trop savantes pour le public ciblé, par des musiques plus directes, mâtinées de références à l'air du temps. C'est là que le bât blesse. De concessions en renoncements le "concert classique de musique électroacoustique" s'effiloche. II est progressivement dénaturé, vidé de son sens, congédié. L'enjeu de cette dérive, c'est effectivement la disparition du concert électroacoustique spéculatif peu rentable au profit du récréatif sensiblement plus payant. La tentation de séduire est forte et le danger bien réel. Mais refouler le concert "savant" dans les studios et les universités accentuerait sa ghettoïsation; ce serait une perte culturelle 
grave car priver le public d'un accès à l'actualité de la recherche vivante aurait pour conséquence de l'infantiliser. Le plus raisonnable semble être une coexistence complémentaire des deux types de manifestation.

Maxime McKinley : Selon vous, l'écoute acousmatique s'applique-t-elle uniquement à la musique issue de la démarche concrète, ou est-il possible d'écouter et de penser toute forme de musique - en particulier la musique instrumentale - de manière acousmatique?

Francis Dhomont : Dans son acception première, l'acousmatique est une attitude d'écoute. Elle est liée à la réduction phénoménologique que Pierre Schaeffer a appliquée à la notion d'uécoute réduite", laquelle consiste à écouter le son pour lui-même, sans tenir compte de son origine. Il est donc effectivement possible d'écouter tout phénomène sonore, donc toute musique, suivant cette intentionnalité. C'est ce que Schaeffer nomme "l'intention d'entendre". Autrement dit, si j'écoute une œuvre orchestrale, je peux m'attacher aux seuls critères typo-morphologiques du sonore et en percevoir les masses, les factures, les entretiens, les grains, les allures, les timbres harmoniques, les profils, etc., au lieu d'identifier mentalement les timbres instrumentaux, les thèmes mélodiques et leurs variations, le jeu des modulations, l'écriture rythmique, ce qui correspond à "l'écoute ordinaire". Mais, sauf à en faire une analyse particulière, cela n'aurait guère de sens pour le mélomane car une œuvre instrumentale n'est pas conçue suivant cette approche. II en va de même pour les œuvres de conception acousmatique que l'on tente d'écouter d'une oreille instrumentale. Le récent ouvrage de Stéphane Roy, L'analyse des musiques électroacoustiques : modèles et propositions ${ }^{2}$, développe ces questions de façon pénétrante.

Maxime McKinley : Lorsque vous entendez des œuvres de nature acousmatique de jeunes compositeurs talentueux, éprouvez-vous une déception, face au fait que l'esthétique acousmatique soit de plus en plus dissoute dans d'autres pratiques électroacoustiques?

Francis Dhomont : Bien sûr, d'autant plus que cette "dissolution" ne donne pas toujours des résultats probants. Mais découvrir un talent acousmatique chez de jeunes compositeurs me confirme, comme je tentais de l'exprimer dans mes premières réponses, que le terreau est toujours riche pour qui sait y planter de bonnes graines et que c'est une contrevérité de le dire épuisé. En ce qui me concerne, il est vrai que je ne doute pas de la nécessité de composer des œuvres purement acousmatiques, c'est d'ailleurs ce qui constitue l'essentiel de ma production. Mais je comprends que de jeunes compositeurs, confrontés à un abondant répertoire, soient tentés de chercher leur propre voie dans divers métissages. Beaucoup, néanmoins, semblent encore trouver leur miel dans une approche orthodoxe, si j'en juge par l'énorme quantité d'œuvres qui parvient aux divers concours de composition acousmatique.

Maxime McKinley : Face aux pratiques électroacoustiques récentes, au nom desquelles Réseaux met un terme à la formule Rien à voir, éprouvez-vous une exaltation quelconque, des espérances?

Francis Dhomont : Il faut toujours faire confiance à ceux qui cherchent et qui mettent en danger leur 
confort pour faire bouger les choses. J'ai donc un a priori favorable, d'autant plus que Réseaux ne nous a jamais déçus jusqu'à présent. C'est donc oui pour les espérances. En revanche, pour l'exaltation je n'en découvre pas beaucoup de motifs autour de moi. La raison en est peut-être que je flaire, derrière certaines tentatives actuelles, l'obsédant désir de plaire, ici et maintenant, plutôt que l'enthousiasme de la recherche. II ne faut pas généraliser, mais succès rapide, popularité, réussite sociale, profit ont tendance à s'imposer comme les valeurs de l'époque. Cet alignement de certains artistes sur le credo actuel de nos sociétés est humainement compréhensible. Mais, au risque de faire vieux, je continue de penser que c'est l'artiste qui peut et doit remettre en question la perversité de l'ordre établi au lieu d'en devenir le débiteur. Au moment où nous assistons partout à une mise à sac de la culture, c'est aux artistes de rompre avec le diktat des marchands plutôt que de faire allégeance. Dans un récent articles, Guy Scarpetta oppose deux idées de la culture "[...] l'une qui la définit comme une mission publique, au service de l'art et de la création; l'autre, qui a intériorisé l'absorption de l'art dans la communication, le spectacle, le festif, le tourisme, l'industrie du divertissement ". C'est parce que cette dernière idée me paraît assez menaçante que mon exaltation reste très modérée.

Je crois que le développement quantitatif de la pratique électroacoustique est irréversible. Une information parue récemment affirmait que les compositeurs électroacoustiques amateurs étaient, en France, quelques dizaines en 1970, un millier en 1980 et qu'ils seraient près d'un million aujourd'hui. Progression exponentielle qui se vérifie probablement dans d'autres pays. Mais quid du qualitatif? Peut-on espérer que l'accroissement pléthorique du nombre de compositions se traduise par un accroissement proportionnel du nombre de chefs-d'œuvre? Ce serait logique. Mais, comme il est également logique qu'il s'accompagne d'une augmentation tout aussi proportionnelle de productions médiocres, le pronostic reste incertain. Le risque, comme cela se constate déjà dans bien des symposiums, c'est que l'avalanche de pièces insignifiantes recouvre les concerts d'une écrasante chape d'ennui et fasse fuir le public des non-spécialistes. C'est pourquoi il me semble vital de retrouver une véritable exigence de qualité. Ce qui a d'ailleurs toujours inspiré la politique des Rien à voir. Je parle, bien sûr, de la qualité intrinsèque des musiques présentées et non du recours à des mises en scènes publicitaires qui ne sont que des cache-misère. À défaut de cette exigence, l'électroacoustique - acousmatique incluse - risque de périr étouffée sous cette prolifération inorganisée, victime de son succès, en quelque sorte, par excès de l'offre mais déficit de la demande.

\section{NOTES}

1. "De la musique et des images", la citation survient à la page 41 de Circuit, vol. 13, $n^{\circ} 3,2003$, p. 41-49.

2. [ndlr] Paru chez l'Harmattan, 2003.

3. "Le grand retour des intermittents du spectacle", Le Monde diplomatique, mai 2004. 